AIAP-1996-043

ITFA-96-10

\title{
Gravitational binding in 4D dynamical triangulation
}

\author{
Bas V. de Bakker* \\ Centre for High Energy Astrophysics \\ Kruislaan 403, 1098 SJ Amsterdam, the Netherlands.
}

\author{
Jan Smit \\ Institute for Theoretical Physics, University of Amsterdam \\ Valckenierstraat 65, 1018 XE Amsterdam, the Netherlands.
}

August 19, 1996

\begin{abstract}
In the dynamical triangulation model of four dimensional euclidean quantum gravity we investigate gravitational binding. Two scalar test particles (quenched approximation) have a positive binding energy, thereby showing that the model can represent gravitational attraction.
\end{abstract}

*email: bas@astro.uva.nl

${ }_{\ddagger}^{\ddagger}$ email: jsmit@phys.uva.nl 


\section{Introduction}

Dynamical triangulation is a discrete approach to the path integral for euclidean quantum gravity in which the euclidean spacetimes are constructed by 'glueing' together geometric objects such as equilateral hypercubes or simplices. Hypercubes were used at first [1] whereas the modern formulation which developed independently out 2D gravity uses simplices. Results of numerical simulations of the commonly used 4D model were first presented in [2, 3]. As a model of gravity dynamical triangulation ought to have a scaling regime where it corresponds to semiclassical Einstein gravity. One wishes to recover classical euclidean spacetimes, Newton's potential and the formation of gravitationally bound states.

It is not clear that a purely euclidean formulation should be able to contain semiclassical gravity, because of the well known divergence related to the unboundedness of the euclidean version of the Einstein-Hilbert action. In a semiclassical evaluation of the euclidean path integral this can be dealt with by deforming the integration over the conformal mode into the imaginary direction of the complex plane $[4$.

The dynamical triangulation formulation is completely regular from the start. The model has a phase transition as a function of a parameter which is proportional to the inverse bare Newton constant: $\kappa_{2} \propto G_{0}^{-1}$. The transition separates a phase with crumpled spacetimes and very high effective dimensionalities, from an 'elongated' phase with effectively two-dimensional spacetimes with characteristics of a branched polymer [5, 6]. Near the transition the model appears to produce classical $S^{4}$-like spacetimes, in an intermediate distance regime, and there is evidence for scaling, suggesting continuum behaviour [5, [7]. In the elongated phase the scaling degenerates into a branched polymer version [6].

It is of great interest to find out if this scaling region can be described by an effective action of the type

$$
S_{\mathrm{eff}}=\int d^{4} x \sqrt{g}\left(\frac{\Lambda}{8 \pi G}-\frac{R}{16 \pi G}+\zeta R^{2}+\cdots\right) .
$$

The branched polymer phase is presumably an expression of the conformal mode instability of the $-R / G$-part in this effective action at scales of order $\sqrt{G}$, while the crumpled phase may correspond to negative $G$.

Recent evidence suggests that the transition is of first order [0, 8], instead of second order as thought previously. The first order nature of the transition need not stand in the way of continuum behaviour as the examples of gaugeHiggs models show. These models have a first order phase transition also in the continuum treatment. For dynamical triangulation we suggested previously

\footnotetext{
${ }^{1}$ We use the phrase 'continuum behaviour' rather than 'continuum limit' to allow for situations in which the lattice distance is very much shorter than any physical distance, but for which the continuum limit would imply unwanted features, such as noninteraction due to triviality (a typical example is the 4D Ising model formulation of $\phi^{4}$ theory).
} 
[5] that continuum behavior may be automatic, as in two dimensions? It is a priori possible that continuum analysis of the effective theory (1) also leads to a

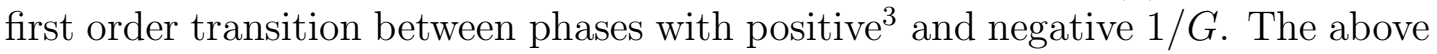
interpretations are very speculative and more analytical as well as numerical work has to be done to establish or reject it.

It would be very interesting if we could measure the attraction of two sources at a fixed distance. This has been pursued in the Regge calculus formulation of simplicial quantum gravity [10]. Such measurements could then be compared with the simple Newtonian law or with quantum corrections to this law, which have for instance been calculated in [11] and [12]. In dynamical triangulation a computation of the gravitational potential appears nontrivial, because it is difficult in a fluctuating spacetime to keep two heavy test masses at a fixed distance.

However, the formation of bound states out of two test particles can be computed in a way that is customary in lattice field theory and we shall report on such computations in this paper. Preliminary results have appeared earlier in [13] and [14]. We see this work as an important ingredient for the interpretation of $4 \mathrm{D}$ dynamical triangulation.

\section{Binding in the continuum}

Consider a free scalar field $\phi$ with bare mass $m_{0}$ in a quantum gravity background. In continuum language, the euclidean action of this system is a sum of a gravitational and a matter part

$$
\begin{aligned}
S & =S[g]+S[g, \phi] \\
S[g] & =\frac{1}{16 \pi G_{0}} \int d^{4} x \sqrt{g}\left(2 \Lambda_{0}-R\right), \\
S[g, \phi] & =\int d^{4} x \sqrt{g}\left(\frac{1}{2} g^{\mu \nu} \partial_{\mu} \phi \partial_{\nu} \phi+\frac{1}{2} m_{0}^{2} \phi^{2}\right),
\end{aligned}
$$

where $\Lambda_{0}$ is the bare cosmological constant, $R$ is the scalar curvature and $G_{0}$ is the bare Newton constant.

We take $\phi$ as a test particle here, i.e. the back reaction of the field $\phi$ on the metric is not taken into account. In lattice QCD this approximation is often

\footnotetext{
${ }^{2}$ We recall also an analogy with $Z(n)$ gauge theories, which have a Coulomb phase for $n \geqslant 5$, with massless photons in a region of parameter space, without the need for tuning to a critical point. Gauge-Higgs models of this type can have furthermore a first order CoulombHiggs transition [9]. The models approach the U(1)-Higgs model as $n \rightarrow \infty$. Because of triviality a continuum limit will be noninteracting, but the models may still be regarded (for finite sufficiently large $n$ ) as a nonperturbative formulation of the abelian Higgs model in the continuum.

${ }^{3}$ In dynamical triangulation the transition actually appears to occur at small positive $1 / G$, since the spacetimes found in its neighbourhood are $S^{4}$-like, cf. sect. 5 .
} 
called the quenched approximation, or valence quark approximation, because it neglects diagrams with internal quark (in our case $\phi$ ) loops. For not too light quark masses it turns out to give good results (for a discussion see e.g. [15]). In dynamical triangulation the inclusion of a scalar field is no problem in principle (and appears to have little influence on the gravity sector of the theory) [16], but the enlargement of parameter space by $m_{0}$ is computationally costly. A continuum calculation of the gravitational attraction of a scalar field in the quenched approximation was carried out in [11.

We will use the following notation for expectation values of an observable $A$. On a fixed background geometry we can average over configurations of the matter field

$$
\langle A\rangle_{\phi}=\frac{\int \mathcal{D} \phi A \exp (-S[g, \phi])}{\int \mathcal{D} \phi \exp (-S[g, \phi])},
$$

and we can average over metrics

$$
\langle A\rangle_{g}=\frac{\int \mathcal{D} g A \exp (-S[g])}{\int \mathcal{D} g \exp (-S[g])},
$$

The quenched expectation value is then

$$
\langle A\rangle=\left\langle\langle A\rangle_{\phi}\right\rangle_{g}
$$

We next consider propagators. In a fixed geometry, the one particle propagator, denoted by $G(x, y ; g)$, is defined as

$$
G(x, y ; g)=\left\langle\phi_{x} \phi_{y}\right\rangle_{\phi}
$$

and the two-particle propagator is simply the square of the one particle propagator. Letting the metric fluctuate, we take the average of the propagators over the different metrics. Because of reparametrisation invariance, the average $\langle G(x, y ; g)\rangle_{g}$ can only depend on whether $x$ and $y$ coincide or not. Therefore, we look at averages at fixed geodesic distance $r$,

$$
G(r)=\left\langle\frac{\int d^{4} x \sqrt{g} G(x, y ; g) \delta(d(x, y)-r)}{\int d^{4} x \sqrt{g} \delta(d(x, y)-r)}\right\rangle_{g}
$$

where $d(x, y)$ is the minimal geodesic distance between $x$ and $y$. By translation invariance, the resulting $G(r)$ is independent of $y$. Similarly, we can define the 
geometry average of the two-particle propagator as

$$
G^{(2)}(r)=\left\langle\frac{\int d^{4} x \sqrt{g} G(x, y ; g)^{2} \delta(d(x, y)-r)}{\int d^{4} x \sqrt{g} \delta(d(x, y)-r)}\right\rangle_{g}
$$

In (9) we first averaged over the volume at distance $r$ from the point $y$ and then averaged over the metrics. Alternatively we can integrate $G(x, y ; g)$ over the volume at distance $r$, average over metrics, and then divide by the same with $G \rightarrow 1$,

$$
G(r)=\frac{\left\langle\int d^{4} x \sqrt{g} G(x, y ; g) \delta(d(x, y)-r)\right\rangle_{g}}{\left\langle\int d^{4} x \sqrt{g} \delta(d(x, y)-r)\right\rangle_{g} \cdot}
$$

A similar ambiguity arises with purely geometric correlators [17. Our physical intuition tends to favour the form (9), and we shall later use its analogue in dynamical triangulation. However, if there is no pair $x, y$ with $d(x, y)=r$ for a given metric $g$ the expression becomes mathematically ill defined, whereas (11) has no such problem. For the pure geometry correlators this does not happen in practise for reasonable $r$ and the difference between (9) and (11) appears to be small [18.

For a massive particle, we expect the propagator (9) to fall off exponentially as

$$
G(r)=Z r^{\alpha} \exp (-m r),
$$

with some power $\alpha$ and renormalised mass $m$, which in general will not equal the bare mass $m_{0}$. This expression neglects finite size effects and should be modified when looking at distances comparable to a typical length scale in the system.

The two-particle propagator will behave similarly as

$$
G^{(2)}(r)=Z^{(2)} r^{\beta} \exp (-M r)
$$

where $M$ is the energy of the two-particle state. If this energy turns out to be less than two times the mass of a single particle, the difference can be interpreted as a binding energy between the particles. This would show gravitational attraction between them.

\section{Propagating in constant curvature}

Since the spacetimes to be used in the binding energy computations have topology $S^{4}$ and the average spacetime is expected to be homogeneous, it will be useful to 
know the properties of scalar field propagators on spaces of constant curvature. To calculate such propagators we have to solve the equation

$$
\left(\square-m^{2}\right) G=0,
$$

with the boundary condition $G \rightarrow\left(4 \pi^{2} r^{2}\right)^{-1}$ as the geodesic distance $r \rightarrow 0$. We assume spherical symmetry, i.e. $G$ depends only on $r$. We need to distinguish three cases: positive, zero and negative curvature. For a space with constant positive curvature, i.e. a four-sphere, equation (14) can be written as

$$
\left(\partial_{x}+3 \cot x\right) \partial_{x} G-m^{2} r_{0}^{2} G=0
$$

where $x=r / r_{0}$, with $r_{0}$ the curvature radius. The substitution $z=\cos ^{2}(x / 2)$, which was used in [19], turns this into a hypergeometric equation,

$$
\left[z(1-z) \partial_{z}^{2}+(2-4 z) \partial_{z}-m^{2} r_{0}^{2}\right] G(z)=0
$$

For numerical evaluation we found it easiest to use a series representation

$$
G(z)=N \sum_{k=0}^{\infty} c_{k} z^{k}
$$

Setting $c_{0}=1$, the differential equation fixes the $c_{k}$ as

$$
c_{k}=\frac{(k+2)(k-1)+m^{2} r_{0}^{2}}{k(k+1)} c_{k-1},
$$

If we demand that the singularity at the origin goes like $\left(4 \pi^{2} r^{2}\right)^{-1}$, this fixes the normalisation to be

$$
N=\frac{m^{2} r_{0}^{2}-2}{16 \pi r_{0}^{2} \cosh \left(\pi \sqrt{m^{2} r_{0}^{2}-\frac{9}{4}}\right)}
$$

One can easily check that this function also conforms to the differential equation at the point opposite the origin, where $x=\pi$. When the mass is so small that $m^{2} r_{0}^{2}<9 / 4$, the above formulas may be analytically continued such that

$$
N=\frac{m^{2} r_{0}^{2}-2}{16 \pi r_{0}^{2} \cos \left(\pi \sqrt{\frac{9}{4}-m^{2} r_{0}^{2}}\right)}
$$

The negative curvature case gives similarly

$$
\left(\partial_{x}+3 \operatorname{coth} x\right) \partial_{x} G-m^{2} r_{0}^{2} G=0 .
$$

Using the substitution $z=\operatorname{sech} x$, we get

$$
\left[\left(1-z^{2}\right) z^{2} \partial_{z}^{2}+\left(-2-2 z^{2}\right) z \partial_{z}-m^{2} r_{0}^{2}\right] G(z)=0 .
$$


In this case we use the series

$$
G(z)=N \sum_{k=0}^{\infty} c_{k} z^{k+\mu}
$$

and the recurrence equation is now

$$
c_{k}=\frac{(k+\mu-2)(k+\mu-1)}{(k+\mu)(k+\mu-3)-m^{2} r_{0}^{2}} c_{k-2} .
$$

We can read off that we have to choose

$$
\mu=\frac{3}{2}+\sqrt{\frac{9}{4}+m^{2} r_{0}^{2}},
$$

to avoid generating negative powers of $z$. Setting again $c_{0}=1$ and demanding that $G$ goes like $\left(4 \pi^{2} r^{2}\right)^{-1}$ for small $r$ gives us the normalisation

$$
N=\frac{\Gamma(\mu / 2) \Gamma(\mu / 2+1 / 2)}{4 \pi^{2} r_{0}^{2} \Gamma(\mu-1 / 2)}
$$

The flat case is the easiest. Using the spherical symmetry results in

$$
\left(\partial_{r}+\frac{3}{r}\right) \partial_{r} G(r)-m^{2} G(r)=0,
$$

which can be directly solved in terms of a Bessel function as

$$
G(r)=\frac{m K_{1}(m r)}{4 \pi^{2} r}
$$

using the condition that $G(r)$ goes like $\left(4 \pi^{2} r^{2}\right)^{-1}$ for small $r$.

We have plotted an example of the three cases in figure 1, using a curvature radius $r_{0}$ of 13 and a mass $m$ of 0.15 . These parameters where chosen for later comparison with dynamical triangulation results.

\section{Dynamical triangulation}

In the dynamical triangulation model of four dimensional euclidean quantum gravity the path integral over metrics on a certain manifold is defined by a weighted sum over all ways to glue four-simplices together at the faces [2, 3]. This idea was first formulated in [1], using hypercubes instead of simplices. In four dimensions the analogue of the continuum gravitational action is

$$
\begin{aligned}
S[g] & =\frac{1}{16 \pi G_{0}} \int d^{4} x \sqrt{g}\left(2 \Lambda_{0}-R\right) \\
& \rightarrow \kappa_{4} N_{4}-\kappa_{2} N_{2},
\end{aligned}
$$




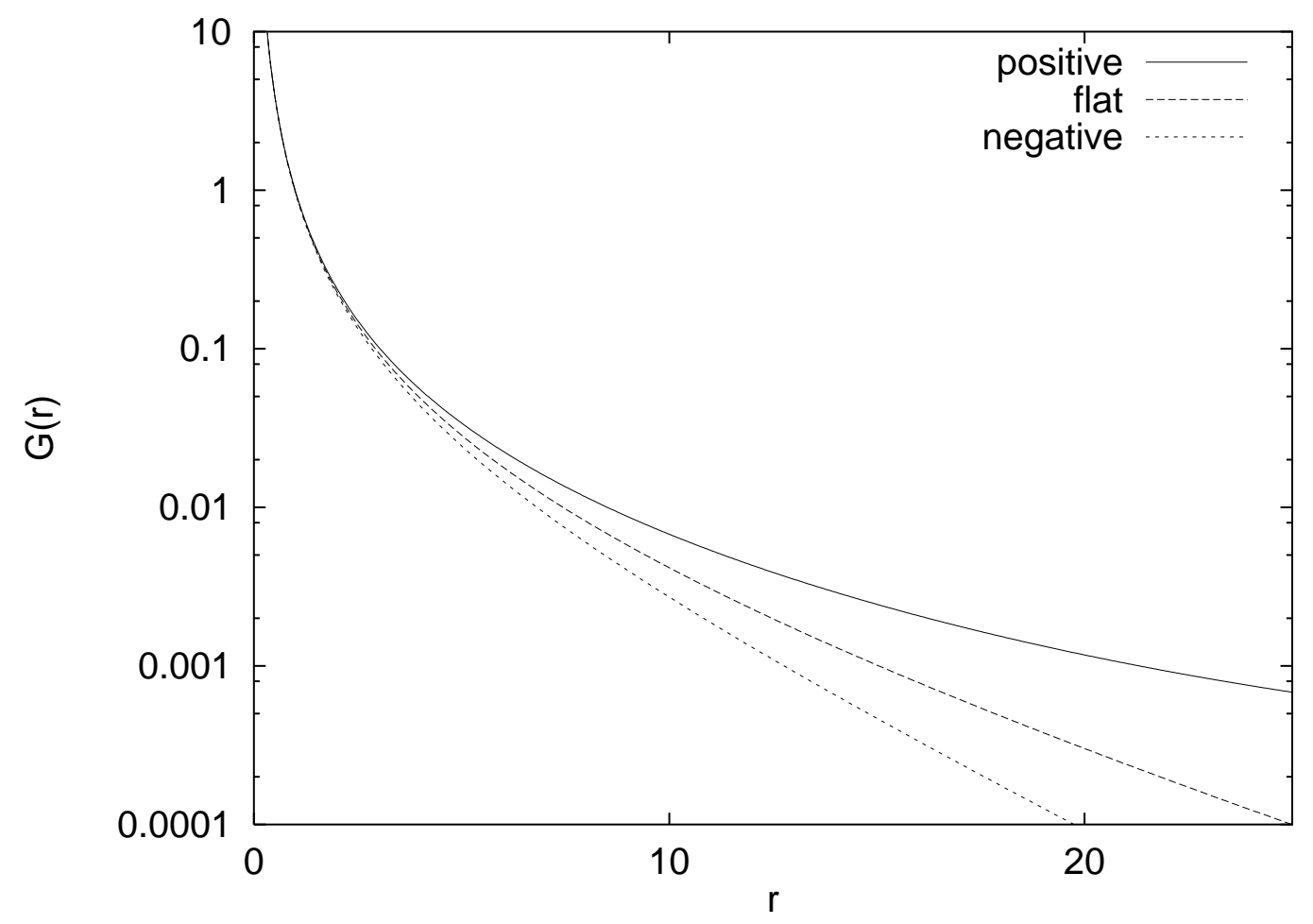

Figure 1: Propagators on spaces with constant curvature $R=12 / r_{0}^{2}$ (upper), $R=0$ (middle) and $R=-12 / r_{0}^{2}$ (lower), with $r_{0}=13$ and $m=0.15$.

where $N_{2}$ and $N_{4}$ are the number of triangles and four-simplices respectively.

The partition function of the model for a fixed volume (fixed number of foursimplices $N_{4}$ ) is given by

$$
Z\left(N, \kappa_{2}\right)=\sum_{\mathcal{T}\left(N_{4}=N\right)} \exp \left(\kappa_{2} N_{2}\right)
$$

The sum is over all ways to glue $N$ four-simplices together, such that the resulting complex satisfies the manifold condition, with some fixed topology which is usually (as well as in this article) taken to be $S^{4}$. The coupling constant $\kappa_{2}$ is proportional to the inverse of the bare Newton constant:

$$
\kappa_{2}=V_{2} / 4 G_{0}
$$

where $V_{2}$ is the area of a triangle.

It turns out that the model has two phases [20, 21, 22, 23]. For low $\kappa_{2}$ the system is in a crumpled phase, where the average number of simplices around a vertex is large and the average distance between two simplices is small. In this phase the volume within a distance $r$ appears to increase exponentially with $r$, a behaviour like that of a space with constant negative curvature. At high $\kappa_{2}$ 
the system is in an elongated phase and resembles a branched polymer. As is the case with a branched polymer, the (large scale) internal fractal dimension is 2 . The transition between the two phases occurs at a critical value $\kappa_{2}^{c}$ which depends somewhat on $N$. The phase transition appears to be of first order [7, 8]. At the transition the spaces behave on the average in several respects like the four dimensional sphere [5]. Some of the evidence for this will be reviewed in the next section.

\section{Spacetimes near the transition}

We have performed numerical simulations of four dimensional dynamical triangulation, according to the partition function (31). We used systems of about 32000 simplices and the topology of the four-sphere. To keep the number of simplices around the desired value, we added a quadratic term to the action as was described in 20, 23, 24].

In ref. [5] we studied the euclidean spacetimes generated in numerical simulations of the model by measuring the number of simplices $N^{\prime}(r)$ at geodesic distance $r$. (The geodesic distance $d_{x y}$ between two simplices with centres $x$ and $y$ is defined as the minimum number of links on the dual lattice between $x$ and $y$.) We fitted $N^{\prime}(r)$ in an intermediate distance regime by a form $c \sin ^{d-1}\left(r / r_{0}\right)$ corresponding to a $d$-sphere of radius $r_{0}$. For $\kappa_{2}$ near the transition $\kappa_{2}^{c}(N)$ this gave $d \approx 4$, which we took as evidence for classical $S^{4}$ behaviour at the distance scale involved. Below the transition (i.e. $\kappa_{2}<\kappa_{2}^{c}$ ) $d$ rises steeply to large values while above the transition $d$ falls rapidly to the branched polymer value $d=2$.

To get a feeling for the geodesic distances which will appear later in binding energy computations, we show in fig. 2 the quantity $N^{\prime}(r)$ for a system of $N=$ 32000 simplices at $\kappa_{2}=1.255$ and 1.259 . The value 1.255 is very near $\kappa_{2}^{c}(N)$ as defined by the position of the maximum in the susceptibility

$$
\frac{\partial^{2} \ln Z\left(N, \kappa_{2}\right)}{N \partial \kappa_{2}^{2}} \approx N\left[\left\langle\frac{N_{2}^{2}}{N_{4}^{2}}\right\rangle-\left\langle\frac{N_{2}}{N_{4}}\right\rangle^{2}\right]
$$

The $\sin ^{d-1}\left(r / r_{0}\right)$-fit gives $d=4.24(4)$ and is seen to be reasonable in the region $6 \leqslant r \leqslant 24$. The value 1.259 is our $\kappa_{2}$ closest to the phase transition for which the data fit the four-sphere also reasonably well with $d=3.67(7)$, in the region $5 \leqslant$ $r \leqslant 25$. The overall shape of $N^{\prime}(r)$ is quite asymmetrical, which is presumably due to branching fluctuations at larger distances.

In [5] we also introduced an effective curvature $R_{\text {eff }}(r)$ to describe the curvature at scales much larger than the lattice scale. The argument $r$ of $R_{\text {eff }}(r)$ is meant to approach zero provided that, and as long as, $R_{\text {eff }}(r)$ is stationary. This does not seem to happen in the elongated phase, while near the transition and in the crumpled phase we found that $R_{\text {eff }}$ had indeed a stationary point (minimum). 


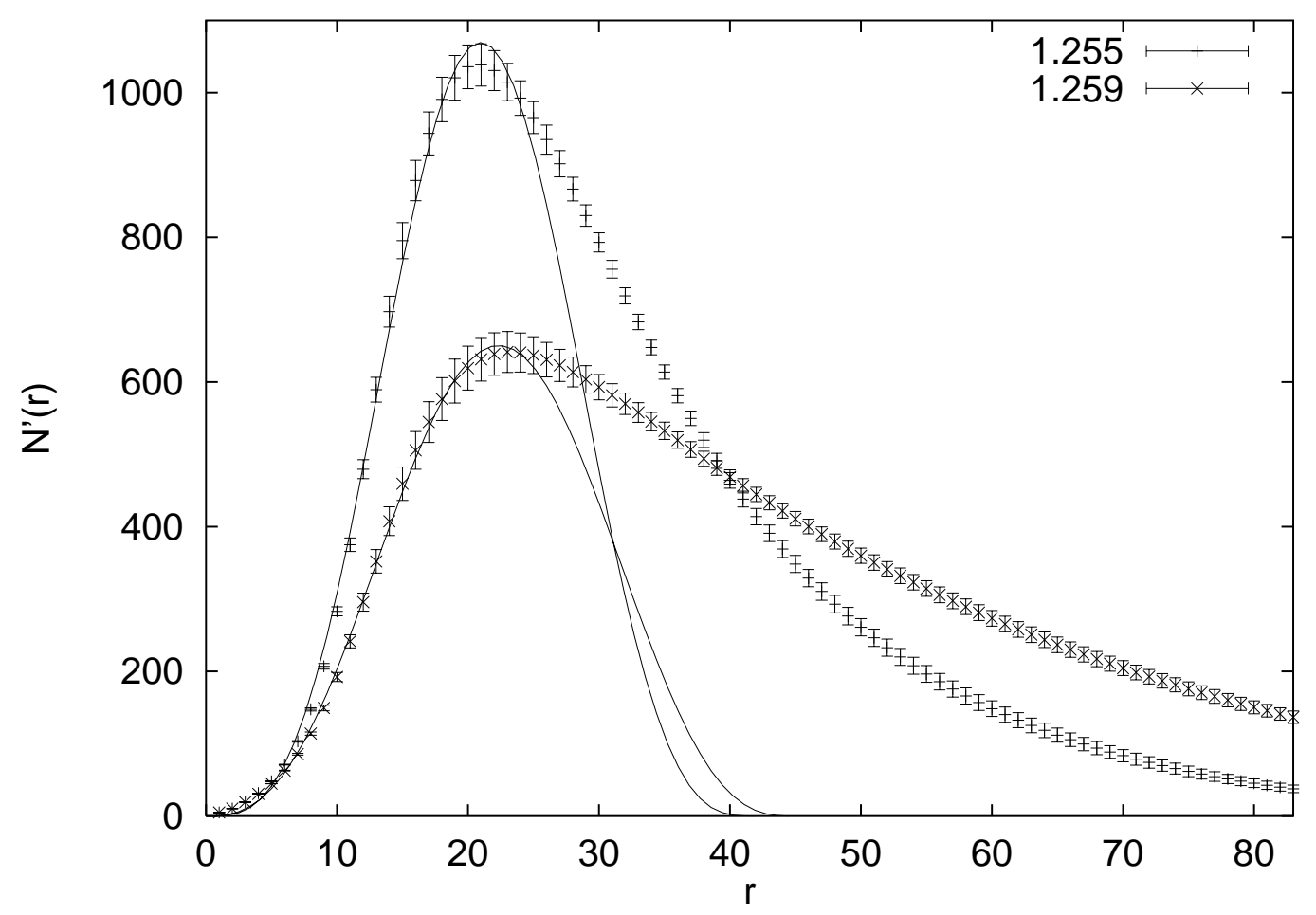

Figure 2: Fits to $N^{\prime}(r)$ of the form $a \sin ^{d-1}\left(r / r_{0}\right)$ near the transition. Upper curve: $\kappa_{2}=1.255, d=4.24(4), r_{0}=13.35(7)$; lower curve: $\kappa_{2}=1.259, d=3.67(7)$, $r_{0}=14.2(2)$.

For smaller $r$-values $R_{\text {eff }}$ rises steeply when $r$ gets smaller. We called this region of large $R_{\text {eff }}$ the 'planckian regime'.

Fig. 3 shows $R_{\text {eff }}$ for $N=32000$ and several values of $\kappa_{2}$. For $\kappa_{2}<1.255$ we see negative curvature at the minimum of $R_{\text {eff }}$. A homogeneous space with constant negative curvature is unbounded, so the maximum in $N^{\prime}(r)$ is evidently due to finite size effects. We take the 'planckian regime' as the small $r$ region, roughly ending at the minimum of $R_{\text {eff }}$. For $\kappa_{2}=1.255$ the effective curvature is nearly zero at the minimum, while positive for larger $\kappa_{2}$. The minimum has roughly turned into an inflection point at $\kappa_{2}=1.259$, and it has disappeared altogether for larger $\kappa_{2}$.

Fig. 目 suggests positive curvature with a curvature radius of $r_{0} \approx 21 /(\pi / 2) \approx$ 13. This corresponds to some average near the minima in fig. 3. The minima will show somewhat smaller curvature: $R_{\text {eff }}\left(r_{\text {min }}\right) \approx 0,0.045$, for $\kappa_{2}=1.255$, 1.259 , respectively. The latter minimum corresponds to a curvature radius $r_{0}=$ $\sqrt{12 / 0.045} \approx 16$, which is not unreasonable compared to the previous 13 .

We conclude that for $\kappa_{2}=1.255-1.259$ the spacetimes are on the average near $S^{4}$ in the distance regime $6 \leqslant r \leqslant 24$. 


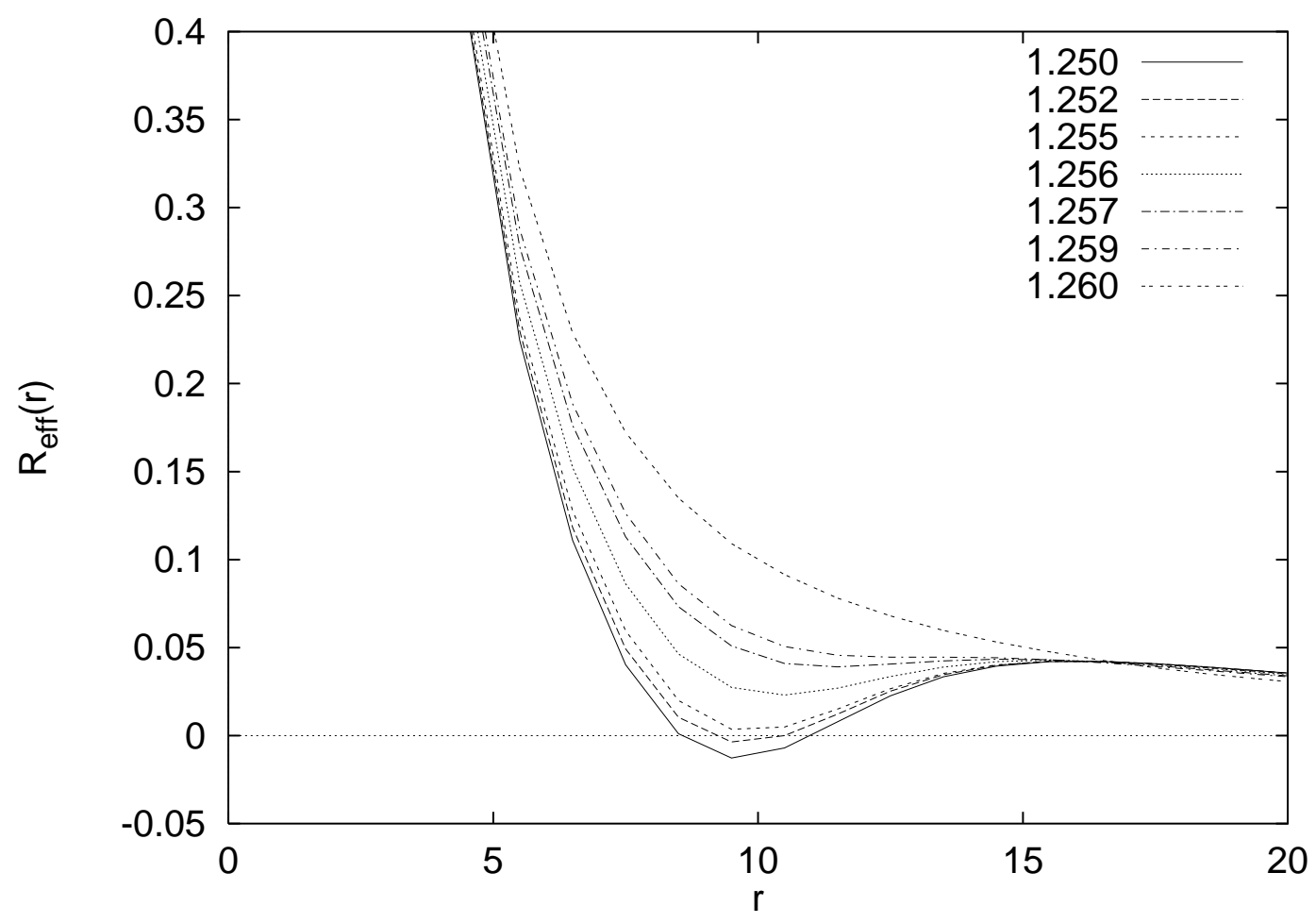

Figure 3: Effective curvature plots for $\kappa_{2}=1.250-1.260$.

\section{Binding in dynamical triangulation}

On each dynamical triangulation configuration we calculated the propagator of the scalar field

$$
G_{x y}=\left(-\square+m_{0}^{2}\right)_{x y}^{-1},
$$

using the algebraic multigrid routine AMG1R5, where $x$ is an arbitrary origin. The discrete Laplacian is defined as

$$
(\square)_{x y}=\left\{\begin{array}{cc}
1 & \text { if } x \text { and } y \text { are nearest neighbours } \\
-5 & \text { if } x=y \\
0 & \text { otherwise }
\end{array}\right.
$$

The 5 in the second line arises as the coordination number of a four-simplex, i.e. a four-simplex has five neighbours.

We can then calculate $G(r)$ and $G^{(2)}(r)$ by averaging $G_{x y}$ respectively its square over all points $y$ at distance $r$ from the origin $x$, and then over origins and configurations,

$$
G(r)=\left\langle\frac{\sum_{y} G_{x y} \delta_{d_{x y}, r}}{\sum_{y} \delta_{d_{x y}, r}}\right\rangle_{g}
$$




$$
G^{(2)}(r)=\left\langle\frac{\sum_{y} G_{x y}^{2} \delta_{d_{x y}, r}}{\sum_{y} \delta_{d_{x y}, r}}\right\rangle_{g}
$$

(we have not indicated the average over origins $x$ ). Notice that (36) corresponds to (9) in the continuum.

To improve the calculation of the binding energy, we can try to use what are called "smeared sources". The use of these smeared sources can improve the data by increasing the contribution of the ground state and decreasing the contribution of the excited states. Instead of using (37), we can calculate $G(r)$ by averaging $G_{x y}$ over all points at distance $r$ from the origin $x$, and only after taking this average, square it for the calculation of $G^{(2)}(r)$ :

$$
G^{(2)}(r)=\left\langle\left(\frac{\sum_{y} G_{x y} \delta_{d_{x y}, r}}{\sum_{y} \delta_{d_{x y}, r}}\right)^{2}\right\rangle_{g} .
$$

This corresponds to taking the propagator from a source that is not a single point, but a complete shell around the origin. Such a source may have a bigger overlap with the ground state wave function and a smaller one with the excited wave functions. For a discussion of the use of these smeared sources in QCD, see e.g. 25.

Because the average of the square of a fluctuating quantity is always greater than the square of its average, it is obvious that $G^{(2)}(r)>G(r)$. This does not yet imply anything about the way they fall off. In particular it is not guaranteed that the energy of the two-particle state is less than twice the energy of the one particle states.

In figure 4 we see the results for four different bare masses. Each pair of lines corresponds to one bare mass. In each pair the upper line is $G^{(2)}(r)$ (using (37)) and the lower line is $G(r)^{2}$ of eq. (36). We used 144 configurations recorded every 5000 sweeps ( 1 sweep $=N$ accepted moves). For each of the masses we used 120 origins per configuration. The coupling constant $\kappa_{2}=1.255$, which is the lower of the two $\kappa_{2}$ values used in the $N^{\prime}(r)$ figure 2 .

There is clearly a difference in slope between the lines in each pair. This shows that the energy of the two particle state is less than two times the mass of a single particle and consequently that there is a positive binding energy between the particles.

Using this data we can measure the renormalised mass $m$, by assuming a long distance behaviour of $r^{\alpha} \exp (-m r)$. The results are (in parenthesis is the value of $\kappa_{2}$ )

\begin{tabular}{c|c|c}
$m_{0}$ & $m(1.255)$ & $m(1.259)$ \\
\hline 0.0316 & 0.14 & 0.12 \\
0.1 & 0.29 & 0.27 \\
0.316 & 0.60 & 0.58 \\
1 & 1.21 & 1.20
\end{tabular}




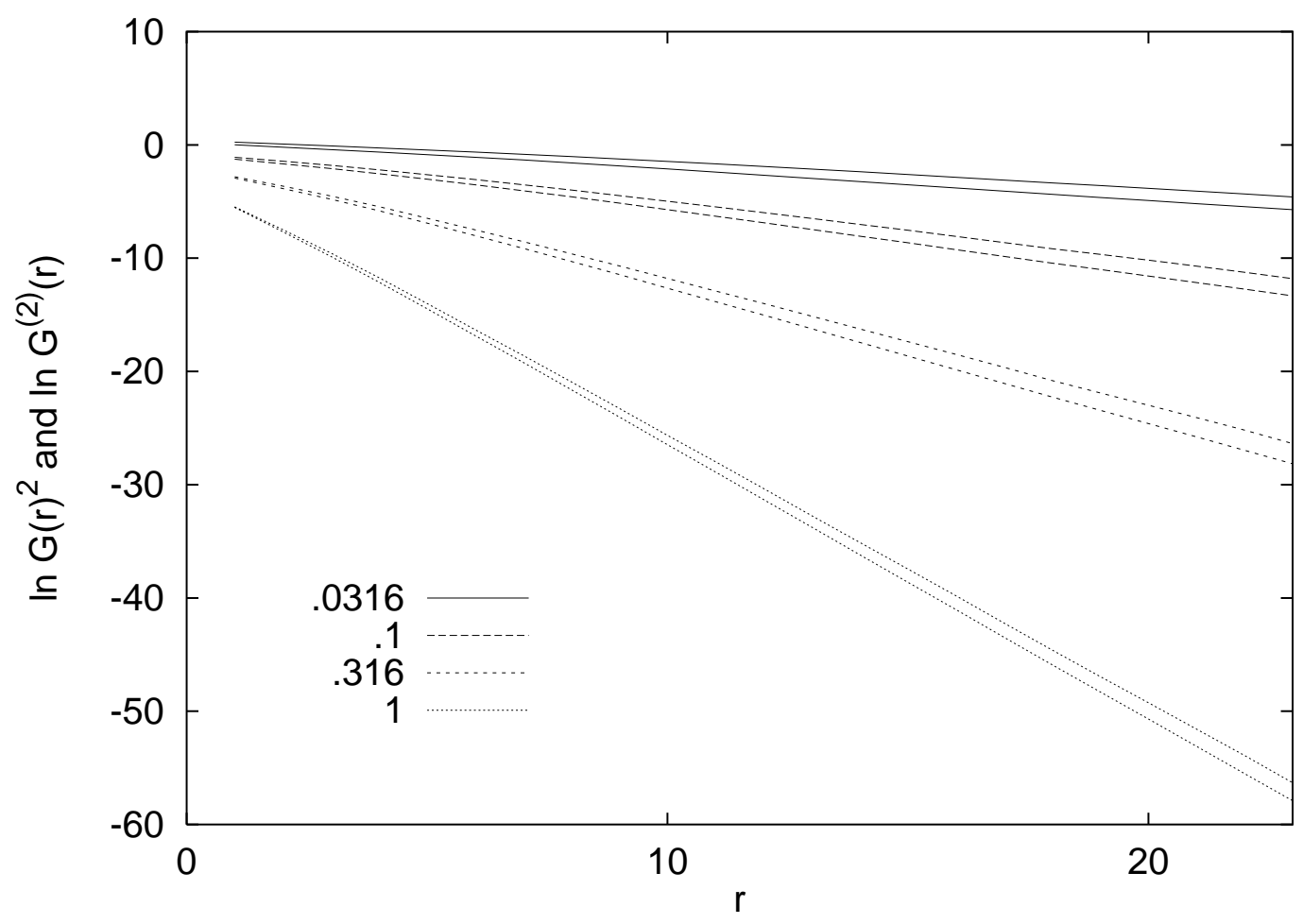

Figure 4: The two-particle propagator and the square of the one particle propagator versus the geodesic distance for four different bare masses $m_{0}$. The vertical scale is logarithmic. $\kappa_{2}=1.255$, which is very close to the transition.

It was argued in [20] that the physical mass should vanish at zero bare mass and that therefore the renormalisation would be only multiplicative. Our data seem to show that the relation is more complicated. Increasing $m_{0}$ by a factor of $\sqrt{10} \approx 3.16$ increases $m$ by a factor of about 2.1 .

Comparing figure 1 with figure $\$$, we see that the long distance behaviour is indeed similar, being an exponential. The finite size effect of the $S^{4}$-like curvature is apparently negligible, except perhaps for the smallest mass. The short distance behaviour is quite different. The propagators in figure 1 curve downward towards the origin, while the free propagators shown in figure 1 curve upwards due to the $1 / r^{2}$ behaviour. The curving downward is unusual, because a propagator is interpreted as a sum of decaying exponentials corresponding to the ground state and various excited states. A closer look shows that the downward curvature occurs for distances smaller than about $r=5$, which is roughly the end of the 'planckian regime' mentioned in the previous section. As we have seen in figure [1, larger positive curvature means that the propagator decreases more slowly. Therefore, such a planckian regime may cause the propagator to decrease less at smaller distances where the effective curvature is large than at the longer distances where the effective curvature is small. 


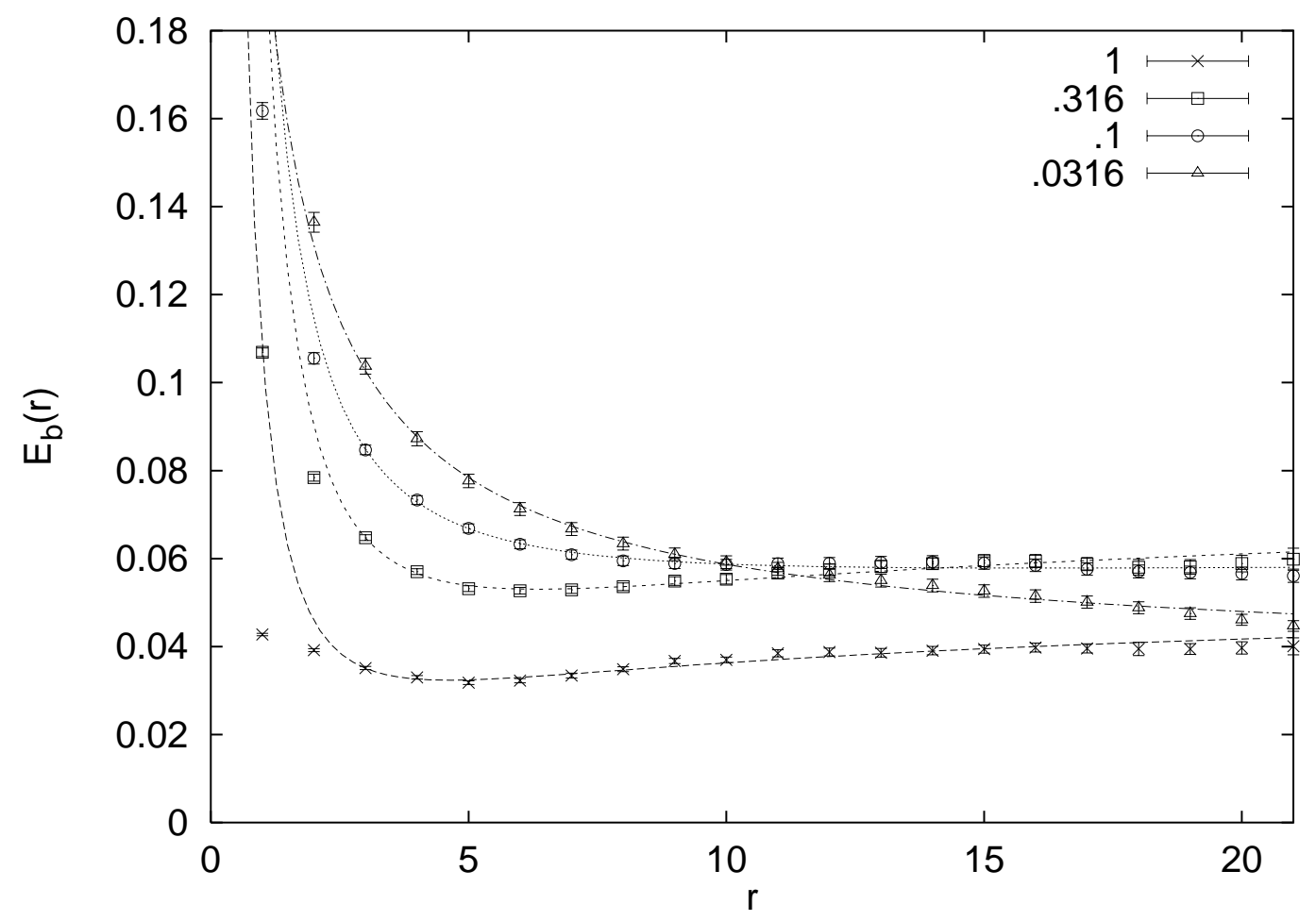

Figure 5: The effective binding energy $E_{b}$ as a function of the geodesic distance for four different bare masses at $\kappa_{2}=1.255$. The lines are fits tot the data with the form (42).

Using these data, we can now estimate the binding energy of the particles. From (12) and (13) we have

$$
\begin{aligned}
E_{b}(r) & \equiv r^{-1} \ln \frac{G^{(2)}(r)}{G(r)^{2}} \\
& \rightarrow E_{b}=2 m-M, \quad r \rightarrow \infty .
\end{aligned}
$$

As we cannot use infinite distances, we will consider the effective binding energy $E_{b}(r)$ for finite $r$ and look whether this expression becomes constant.

Figure 5 shows this quantity as a function of the geodesic distance, using the smeared estimator (38) for $G^{(2)}(r)$. The four curves again correspond to the four different bare masses in figure 6 . To avoid the correlations between origins on the same configuration and between points at the same distance of such an origin influencing the error bars, we first averaged all the measurements of each configuration and used a jackknife method on these averages to calculate the error bars.

It is clear that the binding energy goes to a non-zero value, with the exception of the lowest mass, where the effective binding energy does not seem to converge 


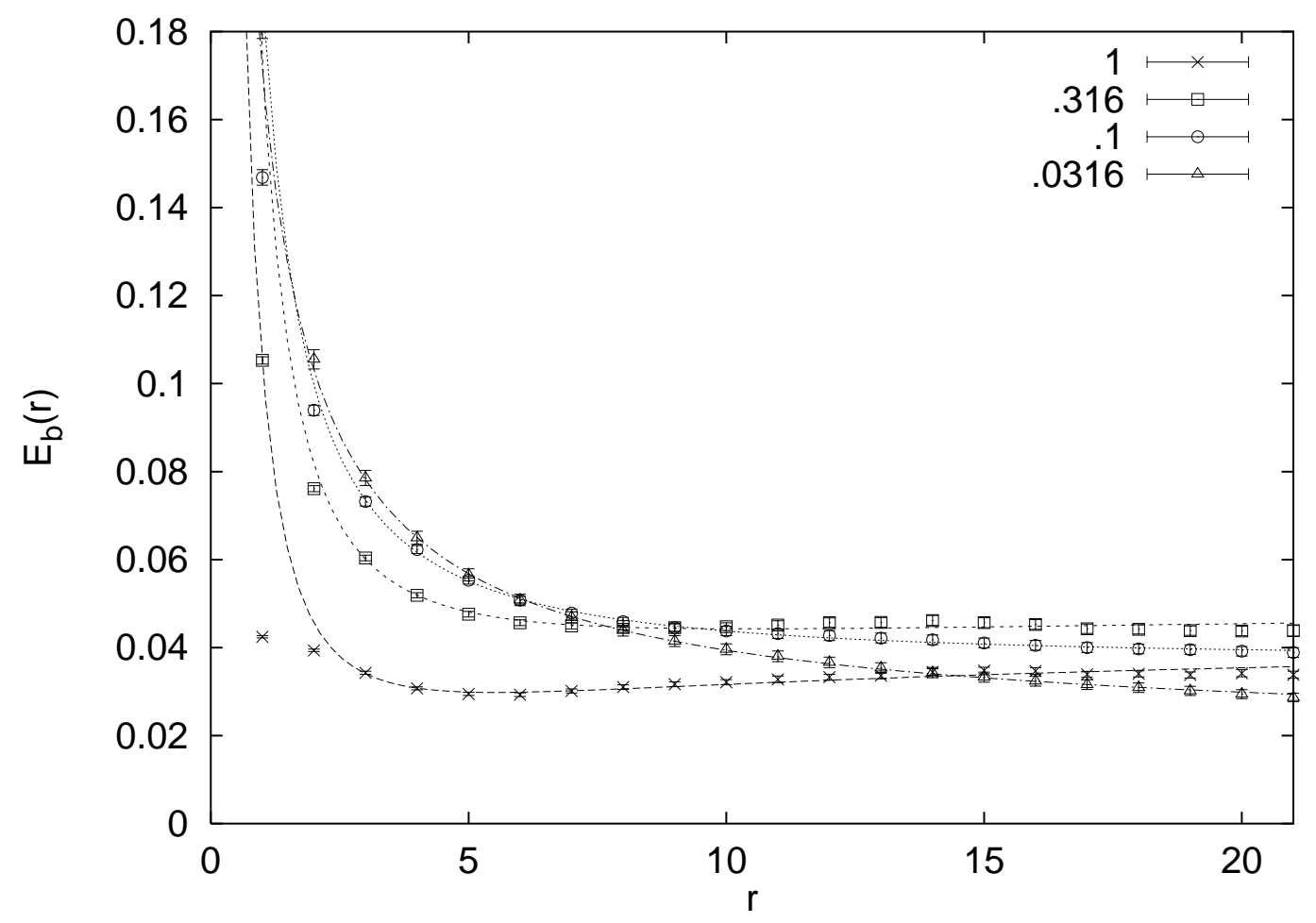

Figure 6: Like figure 5, but with $\kappa_{2}=1.259$.

within the limited distance range. We chose not to display distances larger than the position of the maximum of $N^{\prime}(r)$ in figure 2 .

Unfortunately, the correlation between the mass and the binding energy does not appear to be strictly positive. The lowest binding energy belongs to the highest mass. We defer a more elaborate discussion to the next section.

Figure 6 shows the corresponding data for $\kappa_{2}=1.259$ which is the higher of the two $\kappa_{2}$ values used in figure 2 for $N^{\prime}(r)$. In this case we used 200 configurations, again with 120 origins per configuration. The data look very similar. Here the ordering of binding energies follows more clearly that of the constituent masses, except for the largest mass $m_{0}=1$.

To see the effect of using smeared sources we show in figure 7 the effective binding energy using the unsmeared estimator (37) for $\kappa_{2}=1.259$, the analogue of figure 6. The use of smeared sources (figure 6 compared to figure 7) does indeed improve the convergence to a definite value and the effective binding energies are generally smaller. In particular the curve for a bare mass of 1 , which keeps going down in figure 5 becomes more horizontal. Only the smallest mass is an exception, where neither case has converged yet in the distance range shown.

To determine the binding energies we have fitted the (smeared) effective $E_{b}(r)$ to the form

$$
E_{b}(r)=E_{b}+(c+\gamma \ln r) r^{-1},
$$




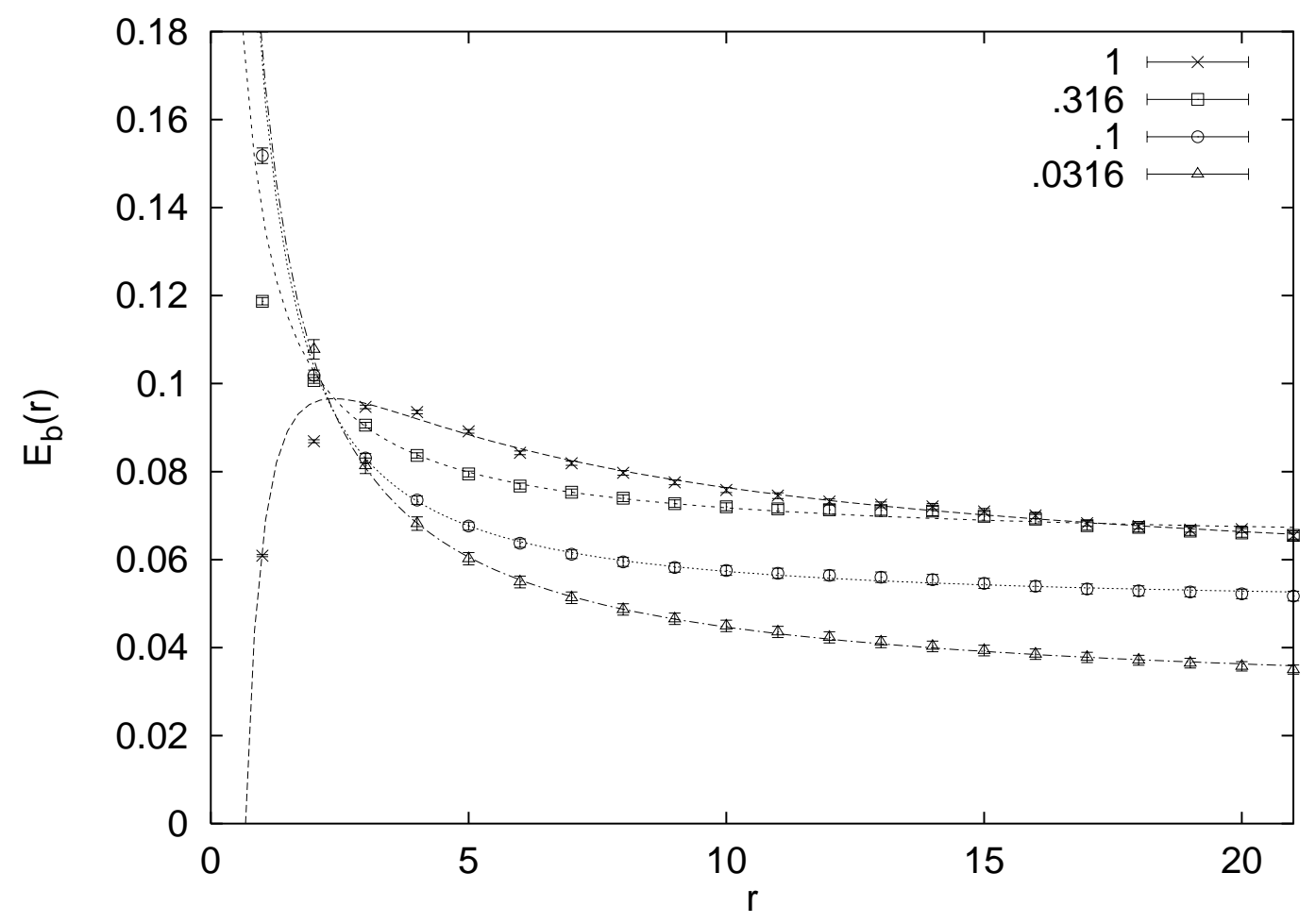

Figure 7: Like figure 6, but with unsmeared sources.

which corresponds to the asymptotic forms (12) and (13) with $c=\ln \left(Z^{2} / Z^{(2)}\right)$ and $\gamma=2 \alpha-\beta$. In general one would expect $c$ to be positive since $\phi^{2}$ will be relatively less effective in creating the two-particle bound state than $\phi$ the single particle state. If the single and two-particle propagators behave sufficiently like a scalar propagator in flat space we would have $\alpha \approx \beta<0$, hence also $\gamma<0$. The results fo the fits in the region $3 \leqslant r \leqslant 20$ (recall $r_{m} \approx 21-22$ ) are shown in the following table.

\begin{tabular}{l|l|l|l|l|l|l}
$m_{0}$ & $E_{b}(1.255)$ & $c(1.255)$ & $\gamma(1.255)$ & $E_{b}(1.259)$ & $c(1.259)$ & $\gamma(1.259)$ \\
\hline 1 & $0.054(1)$ & $0.053(5)$ & $-0.100(7)$ & $0.045(1)$ & $0.059(4)$ & $-0.084(6)$ \\
0.316 & $0.078(2)$ & $0.134(9)$ & $-0.16(1)$ & $0.053(1)$ & $0.19(7)$ & $-0.089(9)$ \\
0.1 & $0.064(2)$ & $0.17(1)$ & $-0.09(1)$ & $0.038(2)$ & $0.15(1)$ & $-0.04(1)$ \\
0.0316 & $0.035(2)$ & $0.17(2)$ & $+0.03(2)$ & $0.019(2)$ & $0.15(2)$ & $+0.02(2)$
\end{tabular}

The signs of $c$ and $\gamma$ appear to follow the above expectations, except for $\gamma$ for the smallest bare mass. The corresponding effective binding energy is still falling for the distances shown and has to be viewed with caution. We cannot improve on this because we do not trust distances much beyond the maximum of $N^{\prime}(r)$ since these run into its asymmetric tail. 


\section{Discussion}

The spacetimes produced by the dynamical triangulation model have semiclassical features near the phase transition. For the binding energy computations we have chosen two values of $\kappa_{2}$ such that the volume at distance $r, N^{\prime}(r)$, behaves like that of a four-sphere, for not too large distances $r$ outside a 'planckian regime'.

Let us briefly recall here our scenario for reducing the lattice distance [5]: increasing $N$ we have to tune $\kappa_{2}$ such that we stay on the same scaling function $\rho=r_{m} N^{\prime}(r) / N$. This can be tried for different starting points $\left(\kappa_{2}, N\right)$, corresponding to different shapes of $\rho$ as a function of $r / r_{m}$. The scaling of $\rho$ has to be carefully re-examined, especially now the phase transition appears to be first order.

The data for a nonzero binding energy are quite convincing and encouraging. For given bare mass $m_{0}$, the resulting renormalized masses $m$ are closer to $m_{0}$ and the binding energies turn out to be smaller for the larger $\kappa_{2}$ value (1.259), than for the smaller value (1.255). This suggests that the renormalized Newton constant $G$ is smaller for the larger $\kappa_{2}$ value, assuming of course $G$ exists and is positive for these $\kappa_{2}$ values. Since $G_{0} \propto 1 / \kappa_{2}$ and one would indeed expect $G$ to decrease when $\kappa_{2}$ increases.

We would like to be able to extract $G$ from the data, for example according to the nonrelativistic formula

$$
E_{b} \equiv 2 m-M=\frac{1}{4} G^{2} m^{5} .
$$

This formula is just the familiar energy $\alpha^{2} m_{\text {red }} / 2$ of the hydrogen atom in the ground state, but with the gravitational parameters substituted as $\alpha \rightarrow G m^{2}$ and the reduced mass $m_{\text {red }} \rightarrow m / 2$. Because the formula (44) is nonrelativistic it may not suffice to fit the data. To get a rudimentary feeling for corrections to (44) we consider the hamiltonian

$$
H=2 \sqrt{m^{2}+p^{2}}-G m^{2} / r .
$$

Replacing $p \rightarrow 1 / r$ and minimising the energy leads to

$$
E_{b}=2 m-2 m \sqrt{1-G^{2} m^{4} / 4}
$$

which suggests that $G m^{2}=2$ has to be considered 'large'.

Unfortunately, the data in figure 5 or 6 show no sign of the $m^{5}$ behaviour of (44), and neither is (46) of any help. Even the largest constituent mass $m \approx 1.2$, which is evidently so large in lattice units that only qualitative conclusions may be drawn from it, leads to a small binding energy. For the lighter constituent masses the binding energy is only modestly dependent on $m$. 
Perhaps the behaviour for the lightest constituent mass hints at a possible interpretation. If we make the bold assumption that the nonrelativistic formula (44) starts making sense for the lightest constituent masses, (39:43) lead to a renormalised Planck length $\sqrt{G}=7.1$ and 7.4 respectively for $\kappa_{2}=1.255$ and 1.259. This appears to contradict the suggestion above that $G$ is smaller for the larger $\kappa_{2}$ value, but the uncertainties in $m$ are greatly magnified by taking its fifth power. The values for $\sqrt{G}$ are reasonable and they are furthermore similar to the (somewhat vague) distance scale the planckian regime ends. On the other hand, the size of a nonrelativistic bound state is of order of $1 / \mathrm{m}$, so if indeed $\sqrt{G} \approx 7$, we should perhaps not be surprised to find odd behaviour for bound state sizes of the order of the Planck length, or constituent masses greater than the Planck mass $1 / \sqrt{G} \approx 0.14$.

Clearly, we are having a problem of separating scales: we would like $\sqrt{G} \ll$ $m^{-1} \ll r_{m}$, where $r_{m}=r_{0} \pi / 2$ is a measure of the size of our $S^{4}$-'universe' (the distance where the volume $N^{\prime}(r)$ is maximal). In our situation, at best, $\sqrt{G} \approx m^{-1}$ and $r_{m} \approx 21 \approx 3 m^{-1}$. The size of our universe is only three times the Planck length. It is essential for a physical interpretation that as the lattices get bigger, the planckian regime and $\sqrt{G}$ shrink in units of $r_{m}$. We found some evidence for this in our scaling analysis [5] where we ventured a scenario in which $\sqrt{G} / r_{m} \rightarrow 0$ because of triviality. This approach to zero might then be only logarithmic, which would make the problem of scale separation severe from the computational point of view. A careful study is needed to clarify these issues. If the separation of scales would not materialize we could still study the continuum behaviour of the complete function $G^{(2)}(r)$ as a welcome addition to the $N^{\prime}(r)$ used so far.

\section{Acknowledgements}

We thank Piotr Białas for useful discussions. The numerical simulations were carried out on the IBM SP1 at SARA and the Parsytec PowerXplorer at IC3A. This work is supported in part by FOM.

\section{References}

[1] D. Weingarten, Nucl. Phys. B210 [FS6] (1982) 229.

[2] J. Ambjørn and J. Jurkiewicz, Phys. Lett. B278 (1992) 42.

[3] M.E. Agishtein and A.A. Migdal, Mod. Phys. Lett. A7 (1992) 1039.

[4] G.W. Gibbons, S.W. Hawking and M.J. Perry, Nucl. Phys. B 138 (1978) 141. 
[5] B.V. de Bakker and J. Smit, Nucl. Phys. B 439 (1995) 239.

[6] J. Ambjørn and J. Jurkiewicz, Nucl. Phys. B 451 (1995) 643.

[7] P. Białas, Z. Burda, A. Krzywicki and B. Petersson, Nucl. Phys. B472 (1996) 293.

[8] B.V. de Bakker, Further evidence that the transition of $4 D$ dynamical triangulation is 1st order, preprint AIAP-1996-040, hep-lat/9603024.

[9] J. Frölich, T. Spencer, Commun. Math. Phys. 83 (1982) 411; V. Alessandrini, Nucl. Phys. B215 (1983) 337; V. Alessandrini, Ph. Boucaud, Nucl. Phys. B225 (1983) 303; K. Jansen, J. Jersak, C.B. Lang, T. Neuhaus, G. Vones, Nucl. Phys. B265 (1986) 129.

[10] H.W. Hamber and R.M. Williams, Nucl. Phys. B 435 (1995) 361.

[11] G. Modanese, Nucl. Phys. B 434 (1995) 697.

[12] H.W. Hamber and S. Liu, Phys. Lett. B 357 (1995) 51.

[13] B.V. de Bakker and J. Smit, Nucl. phys. (Proc. Suppl.) 34 (1994) 739.

[14] B.V. de Bakker and J. Smit, Nucl. Phys. (Proc. Supppl.) 47 (1996) 613.

[15] S.R. Sharpe, in: CP Violation and the limits of the Standard Model, ed. J.F. Donoghue (World Scientific, 1995) p. 377.

[16] J. Ambjørn, Z. Burda, J. Jurkiewicz and C.F. Kristjansen, Phys. Rev. D 48 (1993) 3695.

[17] B.V. de Bakker and J. Smit, Nucl. Phys. B 454 (1995) 343.

[18] P. Białas, private communication.

[19] I. Antoniadis and E. Mottola, J. Math. Phys. 32 (1991) 1037.

[20] M.E. Agishtein and A.A. Migdal, Nucl. Phys. B385 (1992) 395.

[21] J. Ambjørn, J. Jurkiewicz and C.F. Kristjansen, Nucl. Phys. B393 (1993) 601.

[22] B. Brügmann, Phys. Rev. D 47 (1993) 3330.

[23] S. Catterall, J. Kogut and R. Renken, Phys. Lett. B328 (1994) 277.

[24] B.V. de Bakker and J. Smit, Phys. Lett. B 334 (1994) 304.

[25] R. Gupta, Nucl. Phys. B (Proc. Suppl.) 17 (1990) 70. 